\title{
Throughput Maximization of Optical LEO-Ground Links
}

\author{
Nicolas Perlot, Tomaso de Cola \\ Institute of Communication and Navigation, DLR, 82234 Wessling, Germany
}

\begin{abstract}
The long-term throughput of optical links between a circular-LEO satellite and a ground station is assessed for systems with fixed and variable data rates. For a fixed data rate, we evaluate the minimum elevation angle above which the link should be established in order to maximize the throughput. The ratio of this optimized constant-data-rate throughput to the throughput of an adaptive transmission with a data rate proportional to the received power is found to depend mainly on the satellite altitude.
\end{abstract}

Keywords: Atmospheric propagation, Optical communication, Satellite communication.

\section{INTRODUCTION}

Free-space optical links offer higher data rates than their radio counterparts and provide an opportunity to satellites, especially Earth-observation satellites, to bring more data to ground [1]. The communication channel of low-Earth-orbit (LEO) up- and downlinks is affected by several factors (e.g., link distance, atmospheric perturbation, Doppler shift, background noise) that are highly dependent on the satellite elevation angle seen from the ground terminal. Because transmitting data at lower elevations is more difficult, a fixed-data-rate system will be designed for operability down to a certain minimum elevation. In that case, there is a compromise between lowering the minimum elevation above which the link can be established and maintaining a high data rate. Under specific conditions, we propose to evaluate the minimum elevation angle that maximizes the throughput of repeated links between a LEO satellite and a ground station. It is also of interest to know how an adaptive data rate can improve the throughput. Indeed, the increased complexity of an adaptive transmission should be counterbalanced by a significant performance enhancement.

After enouncing the main assumptions underlying the analysis, the normalized throughput is evaluated in Section 3 for a fixed data rate and in Section 4 for a variable data rate. Finally, the significance of the derived formulas is discussed in Section 5.

\section{GENERAL ASSUMPTIONS}

Here is a list of the assumptions underlying our analysis.

(i) The satellite has a low circular orbit and a uniform position probability on its orbital sphere (at least for the orbit region that is visible from the ground station). In Figure 1, the accuracy of this assumption is shown for the case of a polar satellite.

(ii) There is no loss due to optical atmospheric turbulence (effective mitigation of turbulence perturbations is assumed).

The following parameters are constant with respect to the link elevation angle:

(iii) Transmit power.

(iv) Transmitter gain. This implies that the beam Rayleigh range is much shorter than the satellite altitude.

(v) Receiver sensitivity in photons per bit. Equivalently, the achievable data rate is proportional to the received power.

(vi) Cloud probability. Equivalently, the probability of having a line of sight is determined only by the satellite orbit with respect to the ground station.

Free-Space Laser Communication Technologies XXIV, edited by Hamid Hemmati, Don M. Boroson, Proc. of SPIE Vol. 8246, 82460V · (C) 2012 SPIE · CCC code: 0277-786X/12/\$18 · doi: 10.1117/12.908682 


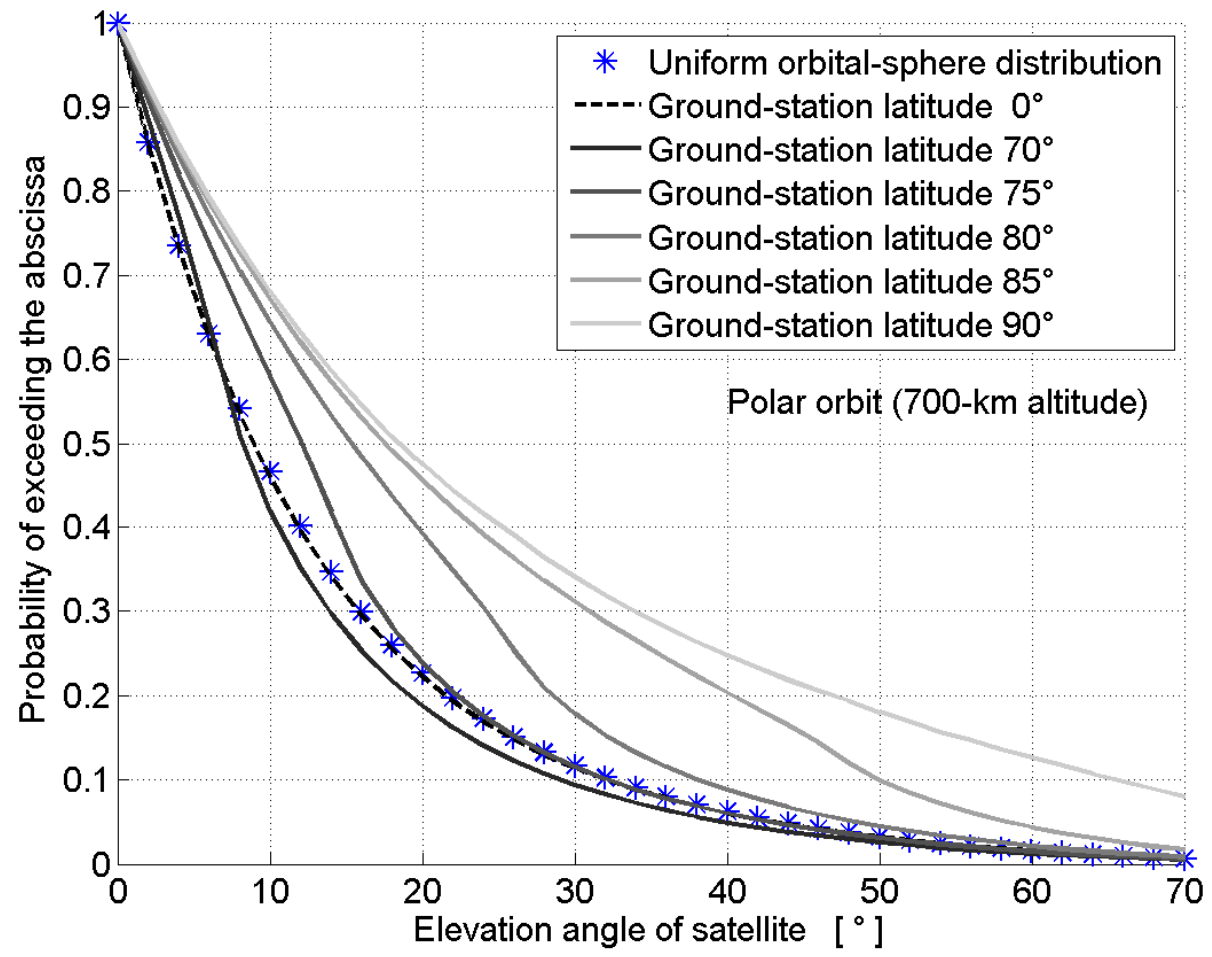

Figure 1: Probability distribution of the satellite elevation (assuming the satellite is above the ground station's horizon). A satellite with polar orbit and $700-\mathrm{km}$ altitude is assumed. For ground-station latitudes between $0^{\circ}$ and $75^{\circ}$, the elevation distribution does not vary much and is close to the distribution produced when the probability of the satellite position is uniform over its orbital sphere.

\section{FIXED DATA RATE}

\subsection{Throughput Definition}

In the context of Section 2, we wish to study the dependence of the long-term throughput on the minimum elevation angle $\alpha$. The throughput is proportional to the link availability rate and to the data rate determined by the received power at the minimum elevation. We thus consider the normalized throughput $T_{f}(\alpha)$ expressed as the product of three functions:

$$
T_{f}(\alpha)=F(\alpha) A_{F S}(\alpha) A_{C S}(\alpha) .
$$

$F(\alpha)$ is the link availability rate; $A_{F S}(\alpha)$ and $A_{C S}(\alpha)$ represent the free-space and the clear-sky attenuations respectively [2]. Applying long-term averages, the function $F(\alpha)$ corresponds to the probability that the elevation of the visible satellite is higher than $\alpha$. Using assumption (i), we find

$$
F(\alpha)=\frac{1}{L^{2}\left(0^{\circ}\right)-L^{2}\left(90^{\circ}\right)}\left[L^{2}(\alpha)-L^{2}\left(90^{\circ}\right)\right]
$$

where $L(\alpha)$ is the link distance as a function of the elevation angle. Noting $H_{\text {sat }}$ the satellite altitude and $R_{e}$ the Earth radius, we have $L\left(90^{\circ}\right)=H_{\text {sat }}$ and $L^{2}\left(0^{\circ}\right)=H_{\text {sat }}^{2}+2 R_{e} H_{\text {sat }}$. Assumption (iv) is generally fulfilled for current satellite terminals [3] and allows to write the normalized free-space attenuation $A_{F S}(\alpha)$ as 


$$
A_{F S}(\alpha)=\frac{L^{2}\left(90^{\circ}\right)}{L^{2}(\alpha)} .
$$

According to Beer-Lambert law, the clear-sky attenuation is given by

$$
A_{C S}(\alpha)=\exp \left[-\int_{0}^{H_{s a t}} a_{e}(h) \frac{d z(h, \alpha)}{d h} d h\right]
$$

where $a_{e}(h)$ is the extinction coefficient in $\left[\mathrm{m}^{-1}\right]$ over the altitude $h$ and $z$ is the propagation-axis distance. The altitude profile of the extinction coefficient influences $A_{C S}(\alpha)$ only at low elevations where the Earth curvature is not negligible. To assess $A_{C S}(\alpha)$ at low elevations, we consider for $a_{e}$ an altitude profile with exponential fall-off:

$$
a_{e}(h)=a_{e, 0} \exp \left(-h / h_{0}\right)
$$

where $a_{e, 0}$ is the extinction coefficient on the ground and $h_{0}$ the extinction characteristic height which equals a few kilometres. Evaluation of Eq. (4) for a zenith link yields a clear-sky attenuation of $A_{C S, 0}=\exp \left(-a_{e, 0} h_{0}\right)$. The positive value in $\mathrm{dB}$ of this attenuation is then

$$
A_{C S, 0, d B}=\frac{10}{\ln (10)} a_{e, 0} h_{0} .
$$

Under the conditions $H_{s a t}=700 \mathrm{~km}, A_{C S, 0, d B}=1 \mathrm{~dB}$ and $h_{0}=3 \mathrm{~km}$, Figure 2 displays the three functions $F(\alpha), A_{F S}(\alpha)$, $A_{C S}(\alpha)$ and their product $T_{f}(\alpha)$. A throughput maximum at a certain elevation angle $\alpha_{0}$ can be observed.

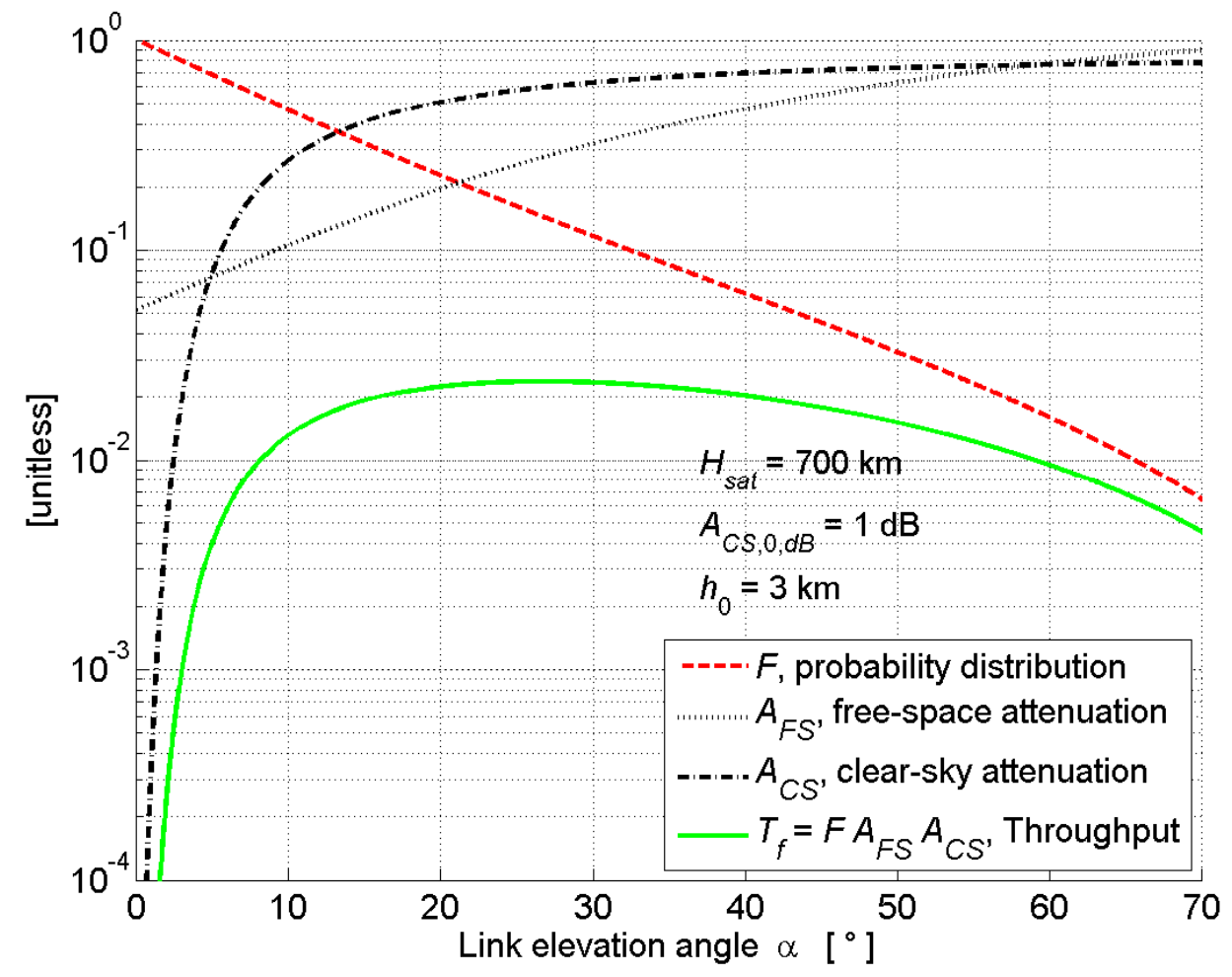

Figure 2: Functions $F(\alpha), A_{F S}(\alpha), A_{C S}(\alpha)$ and their product $T_{f}(\alpha)$ under the conditions $H_{s a t}=700 \mathrm{~km}, A_{C S, 0, d B}=1 \mathrm{~dB}$ and $h_{0}=3 \mathrm{~km}$. 


\subsection{Optimal Minimum Elevation}

To find the optimal minimum elevation $\alpha_{0}$, we first look for the optimal maximum link distance $L\left(\alpha_{0}\right)$ which is found by solving $d T_{f} / d L=0$. To simplify this complex equation, we first neglect the Earth curvature for the clear-sky attenuation, so that Eq. (4) becomes

$$
A_{C S}(L) \approx \exp \left[-\frac{a_{e, 0} h_{0}}{H_{s a t}} L\right]
$$

$d T_{f} / d L=0$ amounts then to a cubic equation which, under the condition $a_{e, 0} h_{0}<27$ (i.e., $A_{C S, 0, d B}<<117$ ), simplifies to

$$
L\left(\alpha_{0}\right) \approx\left(\frac{2}{a_{e, 0} h_{0}}\right)^{1 / 3} H_{s a t} .
$$

From Eq. (8), we deduce $\sin \alpha_{0} \approx\left(\frac{1}{2} a_{e, 0} h_{0}\right)^{1 / 3}$ which, using Eq. (6), yields the following approximation:

$$
\alpha_{0} \approx 0.49 A_{C S, 0, d B}^{1 / 3} .
$$

This optimal minimum elevation also determines the data rate to be implemented. We define the normalized data rate $B_{0}$ as the power attenuation at $\alpha_{0}$ :

$$
B_{0} \equiv A_{F S}\left(\alpha_{0}\right) A_{C S}\left(\alpha_{0}\right)
$$

On the left y-axis of Figure 3, the optimal minimum elevation as given by Eq. (9) is compared to the exact computation. The exact curves are computed for $H_{\text {sat }}=300 \mathrm{~km}$ and $3000 \mathrm{~km}$, showing that the approximation is effective for any LEO satellite altitudes under a wide range of clear-sky attenuations. The extinction characteristic height $h_{0}$ is not specified because $h_{0}$ variations of few $\mathrm{km}$ did not lead to a visually significant variation of the exact curves, even at low $\alpha_{0}$ values. On the right y-axis of Figure 3, the normalized data rate $B_{0}$ is shown.

When the zenith attenuation increases above $A_{C S, 0, d B} \approx 1 \mathrm{~dB}$, we observe a decrease of $B_{0}$, i.e., we observe a weaker received power at the new optimal minimum elevation even though this elevation is higher. Based on the result of Eq. (8) , the optimized fixed-data-rate throughput defined by

$$
T_{f, 0} \equiv F\left(\alpha_{0}\right) A_{F S}\left(\alpha_{0}\right) A_{C S}\left(\alpha_{0}\right) .
$$

can then be written as

$$
T_{f, 0} \approx \frac{H_{s a t}}{2 R_{e}}\left[1-\left(\frac{1}{2} a_{e, 0} h_{0}\right)^{2 / 3}\right] \exp \left[-2^{1 / 3}\left(a_{e, 0} h_{0}\right)^{2 / 3}\right] .
$$




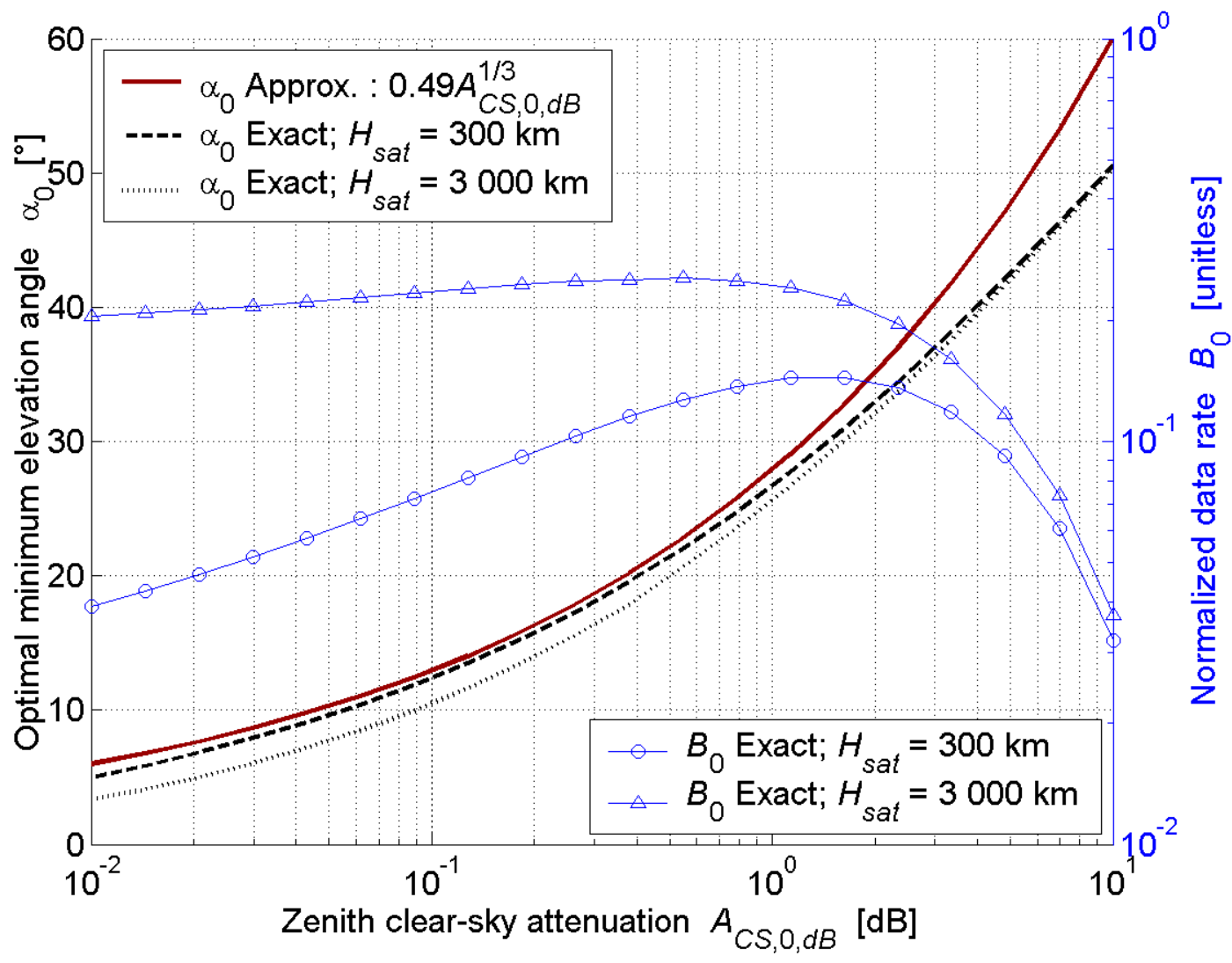

Figure 3: Optimal minimum elevation $\alpha_{0}$ (left y-axis) and associated normalized data rate $B_{0}$ (right y-axis) versus zenith clear-sky attenuation $A_{C S, 0, d B}$. The $\alpha_{0}$ approximation formula is compared to exact values at two different satellite altitudes $H_{\text {sat }}=300 \mathrm{~km}$ and $3000 \mathrm{~km}$.

\section{VARIABLE DATA RATE}

We now consider a variable data rate that is ideally adjusted to the received power. The throughput is then proportional to the received power averaged over its probability of occurrence. Integrating more conveniently over the distance $L$ than over $\alpha$ and noting $f(L) \equiv d F(L) / d L$ the probability density function of the satellite position identified by the link distance $L$, we define the normalized throughput $T_{v}$ according to

$$
T_{v}=\int_{L\left(0^{\circ}\right)}^{L\left(0^{\circ}\right)} f(L) A_{F S}(L) A_{C S}(L) d L .
$$

Introducing $f(L)=2 L /\left(R_{e} H_{\text {sat }}\right)$ as well as Eqs. (3) and (7), we obtain [4]:

$$
T_{v} \approx \frac{H_{s a t}}{R_{e}}\left[\mathrm{E}_{1}\left(a_{e, 0} h_{0}\right)-\mathrm{E}_{1}\left(a_{e, 0} h_{0} \sqrt{1+\frac{2 R_{e}}{H_{s a t}}}\right)\right]
$$

where $\mathrm{E}_{1}(\cdot)$ is the exponential integral. Under the condition $a_{e, 0} h_{0}<<1$, the ratio of Eq. (14) to Eq.(12) yields

$$
\frac{T_{v}}{T_{f, 0}} \approx \ln \left(1+\frac{2 R_{e}}{H_{s a t}}\right)
$$


Figure 4 shows the throughputs $T_{f, 0}$ and $T_{v}$ as a function of the attenuation $A_{C S, 0, d B}$.

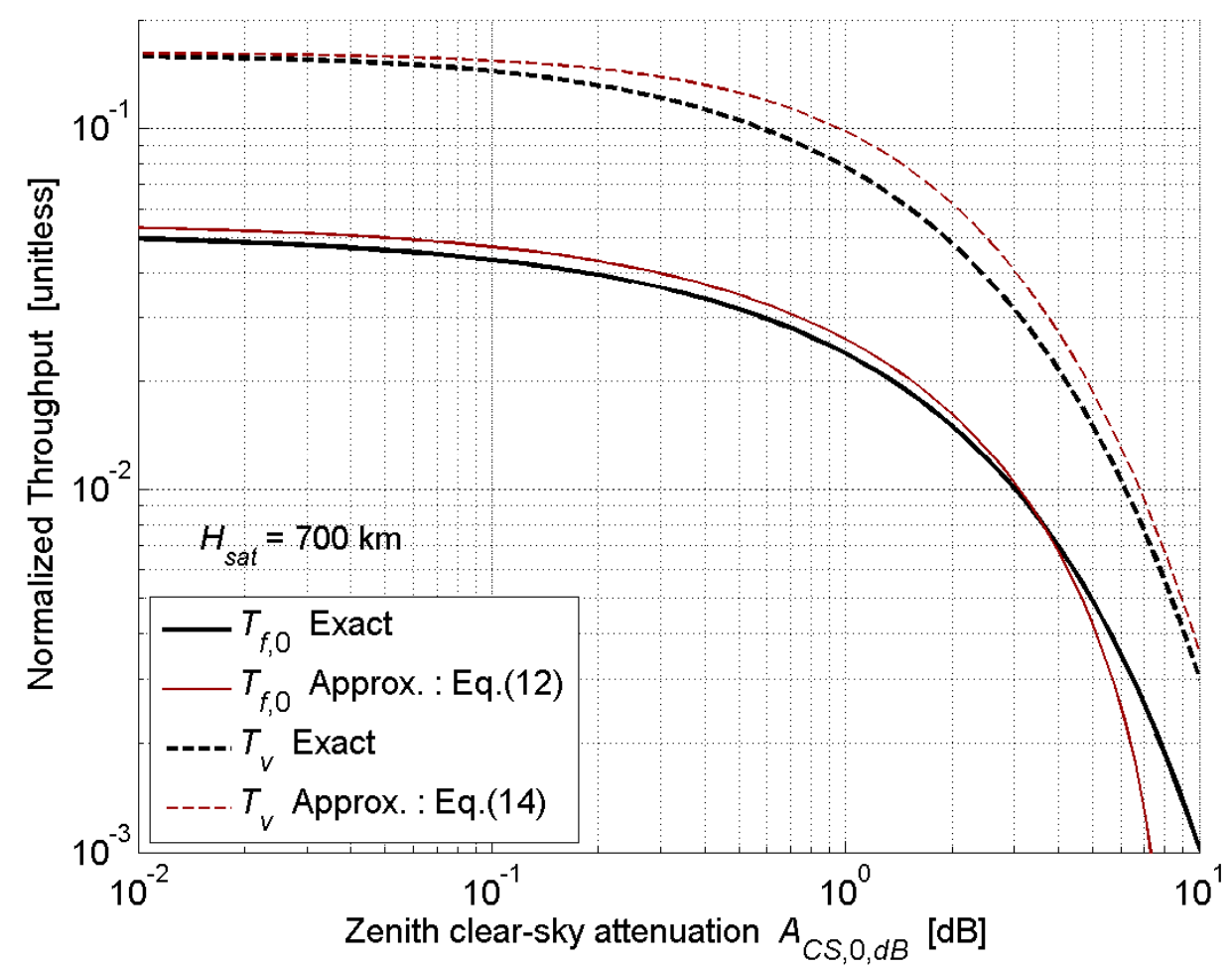

Figure 4: Normalized throughputs for fixed and variable data rates as a function of zenith clear-sky attenuation $A_{C S, 0, d B}$. Exact computations are compared to approximate formulas. Satellite altitude is set to $H_{\text {sat }}=700 \mathrm{~km}$

\section{DISCUSSION}

For a fixed-data-rate system, the minimum elevation that maximizes the throughput is mainly a simple function of the clear-sky attenuation at zenith and therefore can be estimated knowing the wavelength and the local atmosphere at the ground station. When the turbulence loss is significant, we expect the optimal minimum elevation to be higher than the one derived here.

The implementation of an ideally adaptive data rate provides a throughput improvement that depends essentially on the satellite altitude. E.g., for $H_{\text {sat }}=700 \mathrm{~km}$, a throughput 3 times higher is expected. This improvement may be too little compared to the higher complexity of the adaptive solution. However, letting the data rate (or the coding rate) vary has an important advantage: it increases the link availability.

Finally, the presented results should be put into perspective as, in real situations, it is more difficult to estimate the optimal data rate because the atmospheric loss (including turbulence loss) at a ground station changes over time. Furthermore, several ground stations are generally used to increase the number of satellite contacts. These stations may have different attenuations hence different optimal data rates.

\section{REFERENCES}

[1] D. Giggenbach, B. Epple, J. Horwath, and F. Moll, "Optical satellite downlinks to optical ground stations and high-altitude platforms,“" in Proceedings of the 16th IST mobile and wireless communications summit 1-5 July, 2007, Budapest, Hungary

[2] S. Karp, R.M. Gagliardi, S.E. Moran, and L.B. Stotts, Optical Channels: Fibers, Clouds, Water, and the Atmosphere, Plenum Press, New York (1988)

[3] M. Gregory, F. Heine, H. Kämpfner, R. Meyer, R. Fields, C. Lunde, "TESAT Laser Communication Terminal Performance Results in 5.6 Gbit Coherent Inter-satellite and Satellite-to-Ground links", International Conference on Space Optics, Rhodes, Greece (2010)

[4] Abramowitz M., Stegun I. A., Handbook of Mathematical Functions, Dover Publications, New York (1972) 